\title{
The Prevalence and Determinants of Freezing of Gait Among Iranian Patients with Parkinson's Disease
}

\author{
Seyed Amir Hassan Habibi ${ }^{1}(\mathbb{D})$, Seyed Soroush Rahmani ${ }^{(i D}$, Sepide Goudarzi $^{2}$ (D),

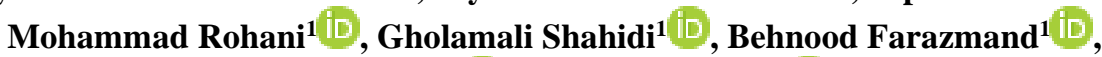 \\ Fereshteh Vosough $^{1}$, Farnaz Vosough $^{1}(\mathbb{D})$, Farhad Modara*3 ${ }^{(D)}$, Fatemeh Mahdizadeh ${ }^{4}(D$
}

1. Dept. of Neurology, Rasoul Akram Hospital, Iran University of Medical Sciences, Tehran, Iran

2. Dept. of Pharmacology and Toxicology, Faculty of Pharmacy, Tehran University of Medical Sciences, Tehran, Iran

3. Dept. of Neurology, Mostafa Khomeini Hospital, School of Medicine, Ilam University of Medical Sciences, Ilam, Iran

4. Neuromuscular Research Center, Semnan University of Medical Sciences, Semnan, Iran

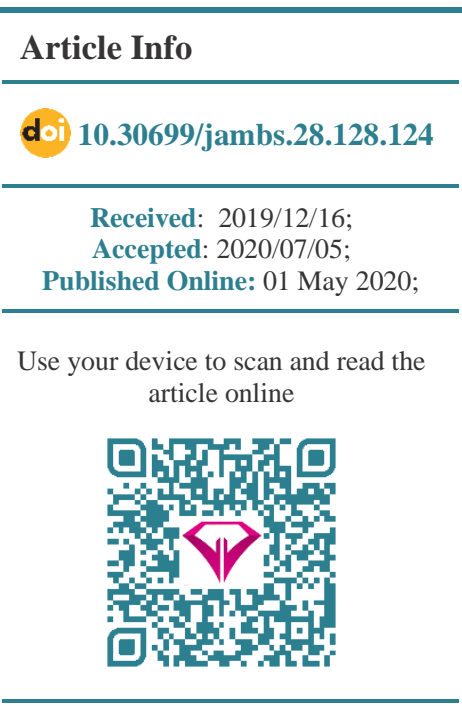

Corresponding Information: Farhad Modara

Dept. of Neurology, Mostafa Khomeini Hospital, School of Medicine, Ilam University of Medical Sciences, Ilam, Iran E-Mail:

Modara-f@medilam.ac.ir

\section{ABSTRACT}

Background \& Objective: Freezing of gait (FOG) is accompanied by various complications, reduced quality of life, and increased risk of mortality and morbidity in patients with Parkinson's disease. The incidence of this disabling phenomenon can be predicted in at least one-third of patients with Parkinson's disease. Therefore, the current study aimed to investigate the prevalence of FOG among Iranian patients with Parkinson's disease and to determine the predictors of this phenomenon in these patients.

Materials \& Methods: This cross-sectional study was performed on 135 patients with Parkinson's disease, referred to Rasoul Akram Hospital, Tehran, Iran. The subjects were selected using the convenience sampling method. FOG was confirmed via clinical examination by two fellows of the movement disorder fellowship program. Variables were evaluated using bivariate and multivariate logistic regression analyses, receiver operating characteristics (ROC) curve analysis, and measurement of the area under the ROC curve (AUC).

Results: Out of 135 patients with Parkinson's disease, 36 (26.7\%) had FOG. Based on the results, FOG had a significantly higher prevalence in older patients, compared to younger patients. According to the results, FOG had no significant relationship with other baseline variables, including gender, disease duration, and disease manifestations. According to the multivariate logistic regression model, advanced age (OR: 0.915; $\mathrm{P}=0.031)$ and longer disease duration $(\mathrm{OR}: 0.992 ; P=0.019)$ predicted the incidence of FOG in the presence of underlying factors.

Conclusion: The prevalence of FOG among patients with Parkinson's disease was estimated at $26.7 \%$, which is within the global range. The results revealed that age and duration of disease were two factors, which could predict the occurrence of FOG with high sensitivity and specificity.

Keywords: Determinants, Freezing of Gait, Parkinson's disease, Prevalence, Risk Factors

\section{Copyright $\odot$ 2020, This is an original open-access article distributed under the terms of the Creative Commons Attribution-noncommercial 4.0 International License which permits copy and redistribution of the material just in noncommercial usages with proper citation.}

\section{Introduction}

Freezing of gait (FOG) is a form of episodic gait disturbance among patients with Parkinson's disease in the absence of a specific cause (1). This condition is also described as a severe and sudden disturbance in walking (2). However, no precise neurological pathogenesis has yet been identified for FOG (3). Evidence suggests that this disorder is not only limited to Parkinson's disease, but responses to dopaminergic and non-dopaminergic drugs may also be the cause (4).

Different hypotheses have been proposed regarding the pathogenesis of FOG. The occurrence of FOG seems to be associated with a disruption in the process of gait coordination at the level of the spinal cord (5). Some researchers have also emphasized on tremor in the legs in a standing position, being related to the impaired coordination of flexor and extensor muscles contraction (6). Another hypothesis suggests the lack of movement automation in the central coordination of muscles being involved in walking (7).

Some imaging studies, such as magnetic resonance imaging (MRI), have also shown a significant reduction in the level of blood oxygenation, especially 
in the vascular pathways of the frontal area $(8,9)$. In general, the lack of coordination in the motor function of the cortex, as well as the spinal cord coordination center, plays a significant role in FOG, especially in Parkinson's disease patients. FOG mainly results in inability to move, disturbance of daily activities, and impaired quality of life (10). The identification of risk factors associated with the occurrence of FOG, which may completely vary across different societies, can contribute to the early diagnosis, prediction, and treatment of this disorder. Therefore, it is essential to first estimate the prevalence and risk factors of FOG in every population.

In the present study, we aimed to determine the prevalence of FOG among Iranian patients with Parkinson's disease and to assess the factors predicting this disabling phenomenon.

\section{Materials and Methods}

This cross-sectional study was performed on patients with Parkinson's disease, referred to Rasoul Akram Hospital, Tehran, Iran, in 2015. The FOG was detected, based on the initial interview with the patient and physical examination in the clinic. Videos were recorded from the patients and checked by two fellows of the movement disorder fellowship program, according to their objective judgment. Also, Parkinson's disease was diagnosed, according to the Movement Disorder Society criteria.

The Student Research Committee of Iran University of Medical Sciences (ethical code: IR.IUMS.rec. 1393.93-02-193-24848) approved the current study. All subjects signed an informed consent form before participating in the study.

\section{Statistical Analysis}

The quantitative variables were expressed as mean and standard deviation, and the categorical variables were described as absolute frequencies and percentages $(11,12)$. Normality of the data was determined by means of the Shapiro-Francia test (13). The comparison of the categorical variables was performed using Chi-square test or Fisher's exact test when more than $20 \%$ of the cells with the expected count of $<5$ were observed. Furthermore, the quantitative variables were compared via the t-test or Mann-Whitney U test. All statistical analyses were performed in SPSS 16.0 (SPSS Inc., Chicago, IL. USA). P-value equal to or less than 0.05 was regarded as statistically significant $(14,15)$.

The variables used to assess the anticipation were gender, disease duration, age, and severity of Parkinson's disease (according to the Unified Parkinson's Disease Rating Scale (UPDRS)).

\section{Results}

Out of 135 patients with Parkinson's disease, 97 (71.9\%) were male. The mean age of the subjects was $57.37 \pm 12.23$ years. Also, the mean duration of the disease was $50.41 \pm 13.44$ months. In terms of clinical manifestations, tremor, rigidity, bradykinesia, and dyskinesia were observed in 100 (74.1\%), 85 (63.0\%), $71(52.6 \%)$, and $20(14.8 \%)$ patients, respectively. Also, 7 (5.2\%) patients reported a history of falling.

Out of 135 patients with Parkinson's disease, 36 (26.7\%) had FOG. The overall prevalence of FOG was significantly higher in older patients, compared to the younger ones (Table 1). Nonetheless, FOG was not associated with other baseline variables, including gender and disease severity. Based on the multivariate logistic regression model (Table 2), advanced age (OR: $0.915 ; P=0.031)$ and longer disease duration (OR: $0.992 ; P=0.019)$ the occurrence of FOG in the presence of an underlying cause was predicted.

According to the area under the receiver operating characteristics curve (AUC) measurements, age was a significant predictor of FOG occurrence (AUC=0.872). The best cut-off point for age to predict FOG was 68.5 years, with sensitivity and specificity of $94.4 \%$ and $59.6 \%$, respectively (Figure 1). The disease duration could also predict FOG (AUC=0.863). The best cut-off point for disease duration was 57.5 months, with a sensitivity of $100 \%$ and specificity of $72.7 \%$ for predicting FOG in patients with Parkinson's disease (Figure 2).

Table 1. Prevalence of freezing of gait based on baseline variables

\begin{tabular}{|c|c|c|}
\hline Factor & Prevalence of freezing of gait & P-value \\
\hline \multicolumn{2}{|l|}{ Gender } & \multirow{3}{*}{0.365} \\
\hline Male & $28(28.9)$ & \\
\hline Female & $8(21.1)$ & \\
\hline \multicolumn{2}{|c|}{ Age group } & \multirow{2}{*}{0.001} \\
\hline$\leq 60$ years & 15 (17.4) & \\
\hline
\end{tabular}




\begin{tabular}{|c|c|c|}
\hline Factor & Prevalence of freezing of gait & P-value \\
\hline$>60$ years & $21(42.9)$ & \\
\hline \multicolumn{2}{|c|}{ Disease duration } & \multirow{3}{*}{0.032} \\
\hline$\leq 50$ months & $25(18.5)$ & \\
\hline$>50$ months & $47(34.8)$ & \\
\hline \multicolumn{2}{|l|}{ Tremor } & \multirow{3}{*}{0.882} \\
\hline Positive & $27(27.0)$ & \\
\hline Negative & $9(25.7)$ & \\
\hline \multicolumn{2}{|l|}{ Rigidity } & \multirow{3}{*}{0.347} \\
\hline Positive & $25(29.4)$ & \\
\hline Negative & $11(22.0)$ & \\
\hline \multicolumn{2}{|c|}{ Bradykinesia } & \multirow{3}{*}{0.113} \\
\hline Positive & $23(32.4)$ & \\
\hline Negative & $13(20.3)$ & \\
\hline
\end{tabular}

Table 2. Multivariate logistic regression model of factors predicting freezing of gait in patients with Parkinson's disease

\begin{tabular}{lllll} 
Index & Odds ratio & Lower limit & Upper limit & P-value \\
\hline Gender & 1.803 & 0.664 & 4.897 & 0.247 \\
\hline Age & 0.915 & 0.845 & 0.992 & 0.031 \\
\hline Duration & 1.092 & 1.014 & 1.176 & 0.019 \\
\hline Constant & 2.284 & --- & --- & 0.524 \\
\hline
\end{tabular}

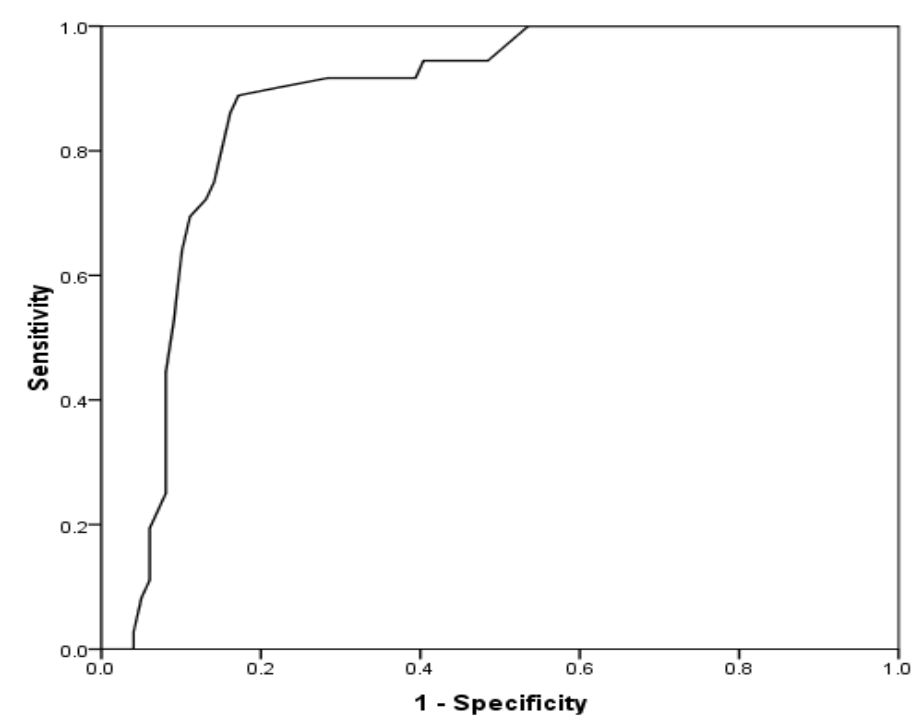

Figure 1. Receiver operating characteristic curve showing the role of patient's age in predicting freezing of gait (area under curve $=0.872, P<0.001)$ 


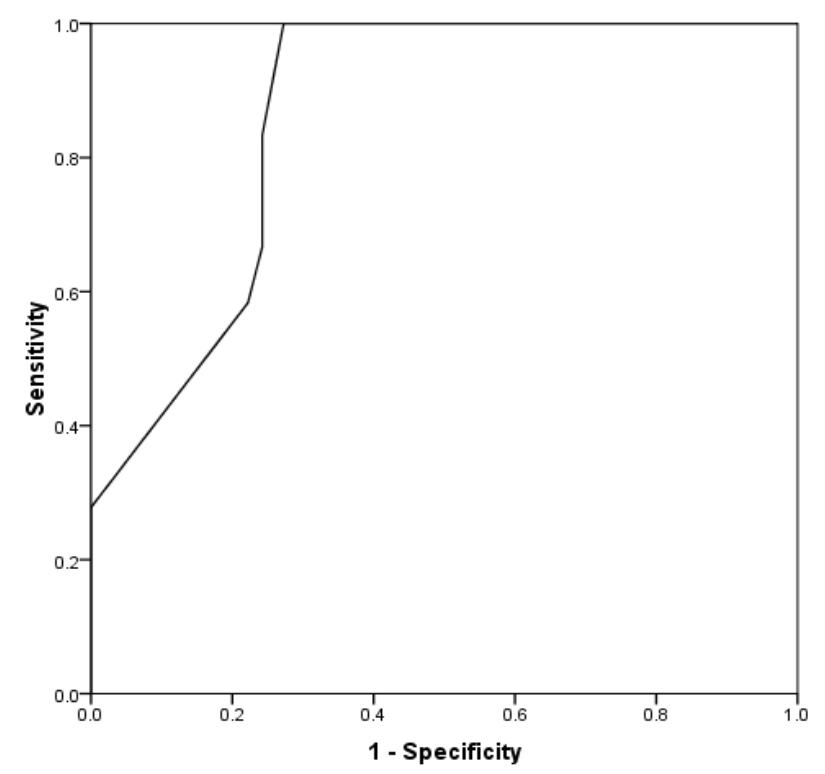

Figure 2. Receiver operating characteristic curve showing the role of disease duration in predicting freezing of gait (area under curve $=0.863, P<0.001$ )

\section{Discussion}

The incidence of FOG is predictable in at least $30 \%$ of patients with Parkinson's disease. This condition is accompanied by various complications, such as walking disorders and disturbances in orientation and balance. Accordingly, this disabling phenomenon can increase the risk of falling and even lead to the development of cognitive, visual, and auditory abnormalities, thereby impairing the quality of life and increasing the risk of mortality and morbidity.

The current study aimed to investigate the prevalence of FOG in Iranian patients with Parkinson's disease and to investigate the predictors of this phenomenon in these patients. The identification of these factors not only can facilitate the early diagnosis of FOG, but also can improve the patient's survival. In the present study, the prevalence of FOG was estimated at $26.7 \%$, which is similar to the rate reported in various studies. In a systematic review, the prevalence of FOG was reported to range from $20 \%$ to $63 \%$ (16).

In a study by et al., the incidence of FOG ranged from $16.1 \%$ to $51.6 \%$, depending on the duration of disease (17). Also, in a study by Amboni et al., an incidence rate of $54.3 \%$ was reported for FOG (18). Moreover, in studies conducted by Forsaa et al. and Contreras et al., the prevalence of FOG was estimated at $38.2 \%$ and $44.4 \%$, respectively $(19,20)$. The discrepancy between the results can be attributed to differences in the sample size or disease severity in these studies.
In the current study, older age and longer disease duration were identified as strong predictors of FOG. The results showed that almost all patients above the age of 68 years, as well as patients with a disease duration of more than five years, were severely affected by FOG. Overall, different predictors have been introduced for FOG in various studies; however, age and duration of disease were found to be the most important ones. In a previous study, the incidence of FOG was $16.1 \%$, $39.5 \%$, and $51.6 \%$ after one, two, and three years of the disease, respectively (17).

Moreover, a previous study examined the association of FOG incidence with a disease duration of more than three years, suburban living, low literacy, akinetic-rigid syndromes, indiscriminate use of levodopa, a high daily dose of levodopa, and failure to use amantadine or dopamine receptor agonists (15). However, in the current study, it was not possible to evaluate the effects of pharmaceutical information and socioeconomic factors on the incidence of FOG due to the lack of adequate patient information.

In consistence with our results, a previous study reported that patients with FOG had a longer duration of disease, a more severe disease, and more disabilities (16). Also, another study reported motor fluctuation and a high dose of levodopa as predictors of FOG; however, these variables were not investigated in our study (17). Overall, in previous studies, patients with FOG and a longer duration of Parkinson's disease had a higher Unified Parkinson's Disease Rating Scale (UPDRS) 
score, higher daily levodopa dose, and more exposure to antimuscarinic drugs (21). The longer duration of Parkinson's disease and higher UPDRS scores were reported as the predictors of FOG. Both factors showed high specificity in the prediction of FOG, despite their low specificity. Therefore, the majority of studies have confirmed the central role of age and duration of disease as two predictors of FOG (19).

Since this is the first study conducted in Iran on FOG, more attention must be paid to this phenomenon, and more appropriate treatments must be prescribed. However, a limitation of this study was that it did not examine the effects of variations in the blood concentration of levodopa or in the level of cognition on the physical status of patients.

\section{Conclusion}

The prevalence of FOG among the Parkinson's disease patients was estimated as $26.7 \%$, in Iran, which is within the global range. The results revealed age and duration of illness as two factors predicting the occurrence of FOG with high sensitivity and specificity. Further studies should be performed to investigate the role of other factors, such as drug and therapeutic approaches, in the prediction of FOG occurrence in our society.

\section{Acknowledgments}

The authors thank the patients and their families for making this study possible through their participation.

\section{Funding}

This research did not receive any specific grant from funding agencies in the public, commercial, or not-forprofit sectors.

\section{Conflict of Interest}

Authors declared no conflict of interest.

\section{References}

1. Giladi N, Nieuwboer A. Understanding and treating freezing of gait in parkinsonism, proposed working defini-tion, and setting the stage.J Movement Disorder Soc. 2008;23(S2):S423-S5. [DOI:10.1002/mds.21927]
2. Fahn S. The freezing phenomenon in parkinsonism. Adv Neurol.1995:53-63.

3. Müller J, Seppi K, Stefanova N, Poewe W, Litvan I, Wenning GK. Freezing of gait in postmortemconfirmed atypical parkinsonism. J Movement Disorder Soc. 2002;17(5):1041-5. [DOI:10.1002/mds.10234]

4. Bonnet A-M, Loria Y, Saint-Hilaire M-H, Lhermitte F, Agid Y. Does long-term aggravation of Parkinson's disease result from nondopaminergic lesions? Neurol. 1987;37(9). [DOI:10.1212/WNL.37.9.1539]

5. Plotnik M, Hausdorff JM. The role of gait rhythmicity and bilateral coordination of stepping in the patho-physiology of freezing of gait in Parkinson's disease. J Movement Disorder Soc. 2008;23(S2):S444-S50. [DOI:10.1002/mds.21984]

6. Hausdorff J, Schaafsma J, Balash Y, Bartels A, Gurevich T, Giladi N. Impaired regulation of stride variabil-ity in Parkinson's disease subjects with freezing of gait. Experiment Brain Res. 2003;149(2):187-94. [DOI:10.1007/s00221-0021354-8]

7. Lewis SJ, Barker RA. A pathophysiological model of freezing of gait in Parkinson's disease. Parkinsonism Relat Disord. 2009;15(5):333-8. [DOI:10.1016/j.parkreldis.2008.08.006]

8. Snijders AH, Leunissen I, Bakker M, et al. Gaitrelated cerebral alterations in patients with Parkinson's dis-ease with freezing of gait. Brain. 2010;134(1):59-72. [DOI:10.1093/brain/awq324]

9. Shine JM, Matar E, Ward PB, et al. Differential neural activation patterns in patients with Parkinson's disease and freezing of gait in response to concurrent cognitive and motor load. PloS one. 2013;8(1):e52602.

[DOI:10.1371/journal.pone.0052602]

10. Mehdizadeh M, Lajevardi L, Habibi SAH, et al. The association between fear of falling and quality of life for balance impairments based on hip and ankle strategies in the drug On-and Off-phase of patients with idio-pathic Parkinson'disease. MJIRI. 2016;30:453.

11. Taghizadeh G, Martinez-Martin P, Fereshtehnejad S$\mathrm{M}$, et al. Psychometric properties of the Berg balance scale in idiopathic Parkinson'disease in the drug offphase. Neurol Sci. 2018;39(12):2175-81. [DOI:10.1007/s10072-018-3570-4]

12. Azad A, Mohammadinezhad T, Taghizadeh G, Lajevardi L. Clinical assessment of activities of daily living in acute stroke: Validation of the Persian version of Katz Index.MJIRI. 2017;31:30. [DOI:10.18869/mjiri.31.30] 
13. Mehdizadeh M, Martinez-Martin P, Habibi S-A, et al. Reliability and validity of fall efficacy scaleinternational in people with Parkinson's disease during On-and Off-Drug Phases. Parkinsons Dis. 2019;2019. [DOI:10.1155/2019/6505232]

14. Mehdizadeh M, Martinez-Martin P, Habibi SA, et al. The association of balance, fear of falling, and daily activities with drug phases and severity of disease in patients with parkinson. Basic Clin Neurosci. 2019;10(4):355-62. [DOI:10.32598/bcn.9.10.295]

15. Taghizadeh G, Martinez-Martin P, Meimandi M, et al. Barthel index and modified rankin scale: psychometric properties during medication phases in idiopathic Parkinson disease. Ann Physic Rehabil Med. 2019. S1877-0657(19)30185-X. [DOI:10.1016/j.rehab.2019.08.006]

16. Bloem BR, Hausdorff JM, Visser JE, Giladi N. Falls and freezing of gait in Parkinson's disease: a review of two interconnected, episodic phenomena. J Movement Disorder Soc. 2004;19(8):871-84. [DOI:10.1002/mds.20115]
17. Zhang $\mathrm{H}$, Yin $\mathrm{X}$, Ouyang $\mathrm{Z}$, et al. A prospective study of freezing of gait with early parkinson disease in Chinese patients. Medicine. 2016;95(26): e4056 [DOI:10.1097/MD.0000000000004056]

18. Amboni M, Stocchi F, Abbruzzese G, et al. Prevalence and associated features of self-reported freezing of gait in Parkinson disease: The DEEP FOG study. Parkinsonism Relat Disorder. 2015;21(6):644-9.

[DOI:10.1016/i.parkreldis.2015.03.028]

19. Contreras A, Grandas F. Risk factors for freezing of gait in Parkinson's disease. J Neurol Sci. 2012;320(12):66-71. [DOI:10.1016/j.jns.2012.06.018]

20. Forsaa E, Larsen J, Wentzel-Larsen T, Alves G. A 12-year population-based study of freezing of gait in Parkinson's disease. Parkinsonism Relat Disorder. 2015;21(3):254-8.

[DOI:10.1016/i.parkreldis.2014.12.020]

21. Perez-Lloret S, Negre-Pages L, Damier P, et al. Prevalence, determinants, and effect on quality of life of freezing of gait in Parkinson disease. JAMA Neurol. [DOI:10.1001/jamaneurol.2014.753]

\section{How to Cite This Article:}

Habibi S A H, Rahmani S S, Goudarzi S, Rohani M, Shahidi G, Farazmand B, et al . The Prevalence and Determinants of Freezing of Gait Among Iranian Patients with Parkinson's Disease. J Adv Med Biomed Res. 2020; 28 (128):138-143

\section{Download citation:}

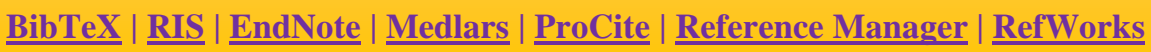

\section{Send citation to:}

(8) Mendeley 2 Zotero (i) RefWorks RefWorks 